\title{
A Case of Multiple Pulmonary Nodules in Association With Pyoderma Gangrenosum
}

\author{
Wei Liu ${ }^{\mathrm{a}}$, Liping Peng ${ }^{\mathrm{a}}$, Shucheng Hua ${ }^{\mathrm{a}}$, b
}

\begin{abstract}
This report describes a patient with pyoderma gangrenosum (PG), in whom there were co-existent lung abnormalities. The patient's CT scan showed peripherally sited multiple pulmonary lesions bilaterally. A lung biopsy showed chronic non-specific inflammatory changes with neutrophil and lymphocyte infiltration which were similar to the skin lesions. This case was diagnosed as multiple aseptic nodules in PG. The pulmonary infiltrative shadows were controlled only with prednisolone treatment. Steroid therapy is considered to be the first choice to control pulmonary lesions of this disease. The main differential diagnosis in these cases is Wegener granulomatosis (WG).
\end{abstract}

Keywords: Pyoderma gangrenosum; Lung multiple nodules

\section{Introduction}

Pyoderma gangrenosum (PG) is a chronic inflammatory cutaneous disease of unknown etiology. Although it is basically considered to be a dermatological disease, its clinical appearance seems to be systemic in some aspects. For example, PG is often associated with chronic inflammatory bowel disease, rheumatoid arthritis, gammopathies or multiple myeloma. However, few cases with pulmonary involvements have been reported. The present case report describes a case of PG with multiple aseptic pulmonary lesions which responded to corticosteroid treatment.

\section{Case Report}

A 63-year-old female retired office worker was admitted to the

Manuscript accepted for publication January 05, 2015

aDepartment of Respiration, the First Hospital of Jilin University, Changchun, China

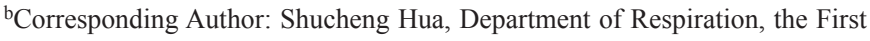
Hospital of Jilin University, Changchun, China.

Email: shuchenghua@eyou.com

doi: http://dx.doi.org/10.14740/jmc2072e authors' respiratory ward in November 23, 2012. She complained of cough and slight chest pain. CT scan showed bilateral infiltrative shadows accompanied by pleural effusion and pleural thickening on the right side (Fig. 1,2). She was treated with oral antibiotics but the shadows did not change.

In previous history, the patient had presented ulcer nodular lesions in the left thigh for 2 years and received several previous treatments, without improvement. The patient has afebrile. There was a dull area on percussion and breath sounds were slightly weak at the right basal area, but crackles were not audible. Laboratory findings revealed leukocytosis (white blood cells $13,200 / \mathrm{mm}^{3}: 75 \%$ neutrophils, no eosinophilia), hemoglobin $13.4 \mathrm{~g} / \mathrm{dL}$, platelet count $461,000 / \mathrm{mm}^{3}$, erythrocyte sedimentation rate $30 \mathrm{~mm} / \mathrm{h}$, C-reactive protein $2.1 \mathrm{mg} /$ $\mathrm{dL}, \operatorname{IgG} 2,530 \mathrm{mg} / \mathrm{dL}$, IgA $418 \mathrm{mg} / \mathrm{dL}$, IgM $197 \mathrm{mg} / \mathrm{dL}$ (normal range of IgG: $885-1,822 \mathrm{mg} / \mathrm{dL}$, IgA: $96-393 \mathrm{mg} / \mathrm{dl}$, IgM: $44-295 \mathrm{mg} / \mathrm{dL})$. Other biochemical blood tests were normal. Rheumatoid factor was negative and both cytoplasmic antineutrophil cytoplasmic autoantibody (C-ANCA) and perinuclear cytoplasmic antineutrophil cytoplasmic autoantibody (P-ANCA) were negative. Tumor markers were negative. Repeated sputum cultures, including those for tuberculosis, were negative. Tomography showed bilateral peripherally located multiple nodular lesions (Fig. 3). A lung biopsy performed from the right middle lobe bronchus showed chronic non-specific inflammatory changes with neutrophil and lymphocyte infiltration, but there was no evidence of bronchiolitis obliterans, granuloma, vasculitis or malignancy. Bronchial washing fluid culture was negative. Percutaneous aspiration by thoracentisis was performed, and fluid cultures including anerobic culture and culture for tuberculosis were negative. Cytologic examination of the lavage fluid showed no malignant cells, only neutrophils and lymphocytes. After (1,200 mg/day) and isepamicin (400 $\mathrm{mg} /$ day) was given intravenously for 10 days, there was no pulmonary improvement.

On December 4, 2013, she started to complain of painful skin ulcerations. The dermatological exam revealed ulcers with discretely elevated erythematous-violaceous borders, necrotic and hemorrhagic base bilaterally in the left thigh (Fig. 3 ). The histopathological exam of the cutaneous lesion showed spongioticfoci, lymphocyte and eosinophil exocytosis in the epidermis and, in the dermis, vascular proliferation and dense mixed inflammatory infiltrate composed of neutrophil and lymphocyte infiltration, without angitis or granuloma, which 


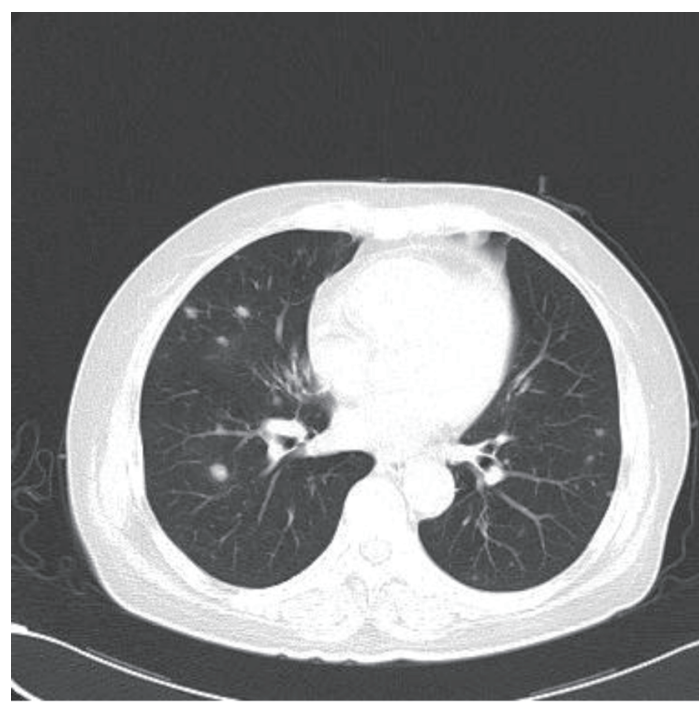

Figure 1. CT scan.

were similar to the lung biopsy (Fig. 4). These macroscopic and microscopic findings demonstrated typical features of PG.

This case was diagnosed as multiple pulmonary nodules in association with PG, oral prednisolone was given at $40 \mathrm{mg}$ / day, and the infiltrative shadows rapidly decreased over a few weeks. The patient was discharged on December 10, 2013 and prednisolone was tapered to $10 \mathrm{mg}$ /day. During the subsequent 6 months, no relapse has been recognized.

\section{Discussion}

PG, first described by Brunsting and O'Leary in 1930, is a rare neutrophil, ulcerative, painful and non-infectious dermatosis, described as having a strong association with ulcerative colitis of unknown pathogenesis [1-3].

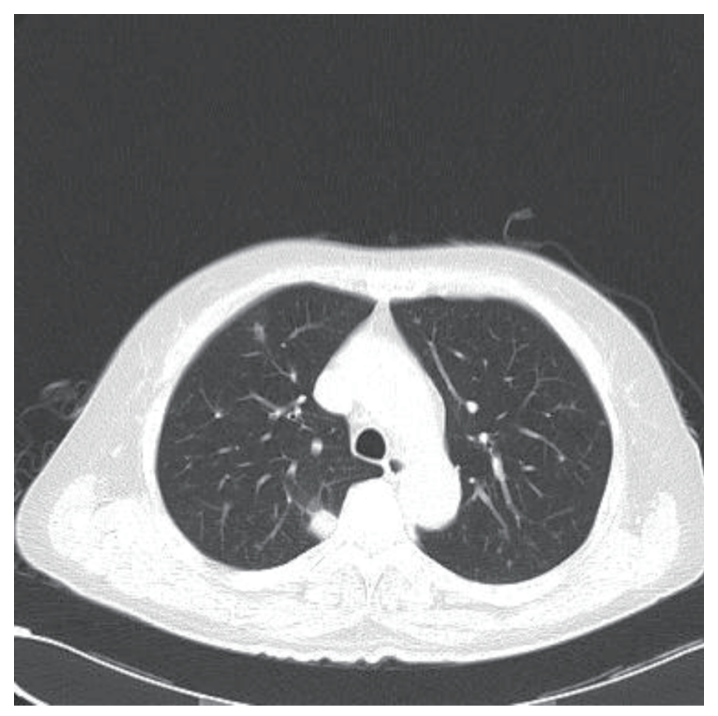

Figure 2. CT scan.

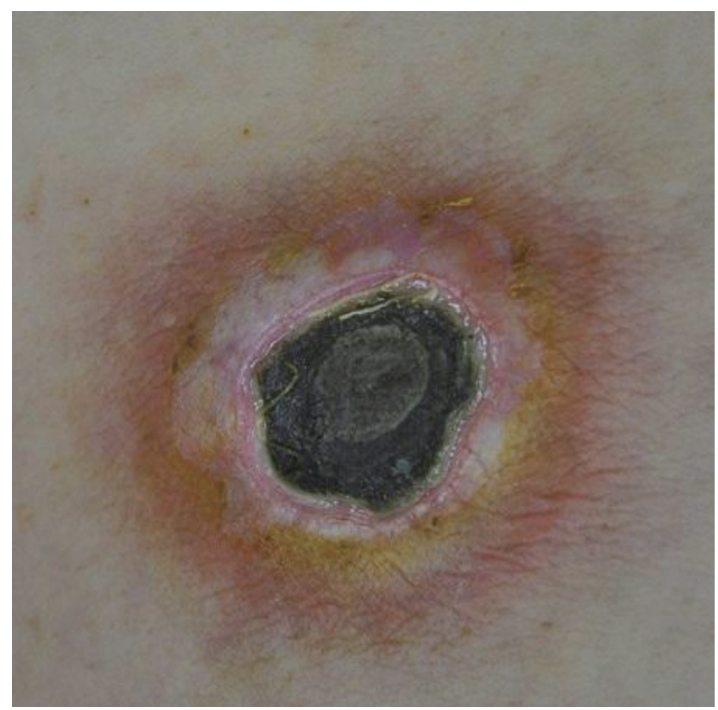

Figure 3. Skin lesion.

Pulmonary involvement of PG is uncommon and has been seldom reported [4-7]. Previously reported histological findings of pulmonary lesions are similar to those of the present case $[4,8-10]$. The lung abscesses were aseptic [4, 8-10]. In the other cases, pulmonary lesions showed a single unilateral opacity on CT scan. The present patient had an unusual form of PG with multiple pulmonary involvements which has not been reported previously.

PG is characterized by an aseptic inflammatory cutaneous disease of unknown etiology. It is associated with basic immunological disorders like Cohn's disease, ulcerative colitis, rheumatoid arthritis, vasculitis, lymphomas, leukemia, dysproteinemia or multiple myeloma [11]. There may be autoimmune factors in the development of the disease process. The previous reported cases with pulmonary lesions also had basic diseases, ulcerative colitis and rheumatoid arthritis. This case

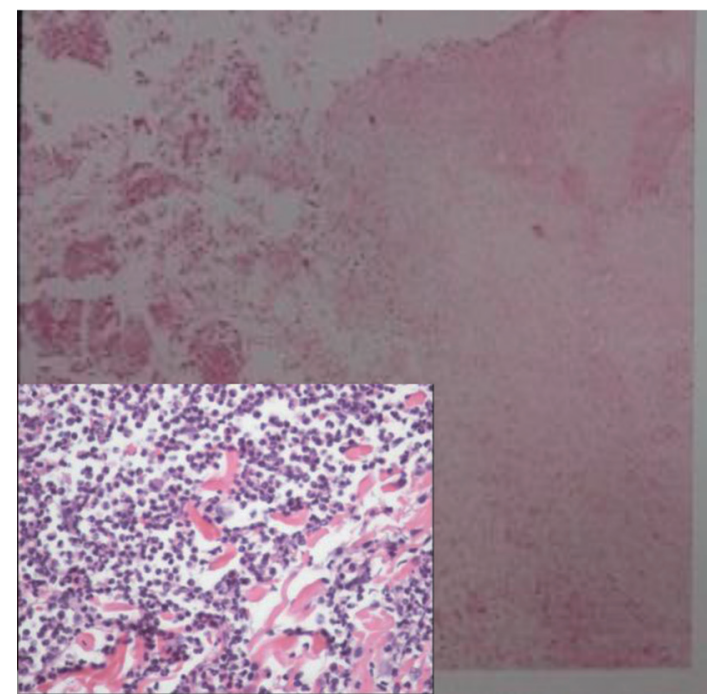

Figure 4. Dermatology pathology. 
showed increase of immunoglobulins, but there were no clinical complications of other immunological disorders [12]. The main differential diagnosis in such cases is Wegener's granulomatosis (WG), which makes the CT-guided lung biopsy necessary $[7,13,14]$. PG and $\mathrm{WG}$, in a limited form, present similarities in the clinical and histopathological presentations $[3,15$, 16]. In lung PG, however, the inflammatory lesions are aseptic, without necrotizing granulomatous inflammation or vasculitis $[15,14,17]$. In addition, the C-ANCA is highly specific for WG, present in $80-90 \%$ of the patients with classical WG and in $55-66 \%$ if those with limited WG.

In this case, the response to steroid therapy was dramatic, as in previously reported cases. Steroid therapy is considered to be the first choice to control pulmonary lesions of PG [7, 14, 17]. Only no more than 12 similar cases have been published in the international literature [14]. It is of fundamental importance to know this clinical association, so little known by dermatologists and radiologists, for more success in the diagnosis, treatment and follow-up of these patients.

\section{Acknowledgement}

The authors would like to thank Prof. Li Pingpeng for her assistance and educational comments.

\section{Conflict of Interest}

The authors declared that there was no conflict of interest in this work.

\section{References}

1. Powell FC, Schroeter AL, Su WP, Perry HO. Pyoderma gangrenosum: a review of 86 patients. Q J Med. 1985;55(217):173-186.

2. Brunsting LA, Goeckerman WH, O'Leary PA. Pyoderma (echthyma) gangrenosum: clinical and experimental observations in five cases occurring in adults. Arch Dermatol Syph. 1930;22:655-680.

3. Berbert AL, Mantese SA, Rocha A, Rezende J, Cunha TF. [Superficial granulomatous pyoderma: report of a case of an uncommon variant of pyoderma gangrenosum]. An Bras Dermatol. 2009;84(3):285-288.

4. Vignon-Pennamen MD, Zelinsky-Gurung A, Janssen F, Frija J, Wallach D. Pyoderma gangrenosum with pulmonary involvement. Arch Dermatol. 1989;125(9):1239-
1242.

5. Vignon-Pennamen MD, Wallach D. Neutrophilc disease: a review of extracutaneous neutrophilic manifestations. Eur J Dermatol. 1995;5:449-455.

6. Brown TS, Marshall GS, Callen JP. Cavitating pulmonary infiltrate in an adolescent with pyoderma gangrenosum: a rarely recognized extracutaneous manifestation of a neutrophilic dermatosis. J Am Acad Dermatol. 2000;43(1 Pt 1):108-112.

7. Kruger S, Piroth W, Amo Takyi B, Breuer C, Schwarz ER. Multiple aseptic pulmonary nodules with central necrosis in association with pyoderma gangrenosum. Chest. 2001;119(3):977-978.

8. Holt PJ, Davies MG, Saunders KC, Nuki G. Pyoderma gangrenosum: clinical and laboratory findings in 15 patients with special reference to polyarthritis. Medicine (Baltimore). 1980;59(2):114-133.

9. McCulloch AJ, McEvoy A, Jackson JD, Jarvis EH. Severe steroid responsive pneumonitis associated with pyoderma gangrenosum and ulcerative colitis. Thorax. 1985;40(4):314-315.

10. Lebbe C, Moulonguet-Michau I, Perrin P, Blanc F, Frija J, Civatte J. Steroid-responsive pyoderma gangrenosum with vulvar and pulmonary involvement. J Am Acad Dermatol. 1992;27(4):623-625.

11. Hickman JG, Lazarus GS. Pyoderma gangrenosum: a reappraisal of associated systemic diseases. Br J Dermatol. 1980;102(2):235-237.

12. Huff JC, Golitz LE, Kunke KS. Intraepidermal neutrophilic IgA dermatosis. N Engl J Med. 1985;313(26):16431645.

13. Field S, Powell FC, Young V, Barnes L. Pyoderma gangrenosum manifesting as a cavitating lung lesion. Clin Exp Dermatol. 2008;33(4):418-421.

14. Batalla A, Perez-Pedrosa A, Garcia-Doval I, GonzalezBarcala FJ, Roson E, de la Torre C. [Lung involvement in pyoderma gangrenosum: a case report and review of the literature]. Actas Dermosifiliogr. 2011;102(5):373-377.

15. Barbato MT, Bakos L, Masiero NCMS, Bolson P. Perfil clinicopatologico dos pacientes com pioderma gangrenoso do Hospital de Clinicas de Porto Alegre (2000-2006). An Bras Dermatol. 2008;83:431-436.

16. Wong YW, Lyon CC, Benbow EW, Bradley BL, Beck MH. Pyoderma gangrenosum in a thoracotomy wound associated with a pulmonary cavitating lesion. Clin Exp Dermatol. 2003;28(3):274-276.

17. Liu ZH, Lu XL, Fu MH, Zhang GY, Liu WD. Pyoderma gangrenosum with pulmonary involvement? Eur J Dermatol. 2008;18(5):583-585. 